\title{
Seleção Ótima do Conjunto de Antenas para um canal MIMO Faixa Larga
}

\author{
Rodrigo F. Souto e Robson D. Vieira
}

\begin{abstract}
Resumo-Neste trababalho, avaliou-se a seleção de antenas para um canal MIMO medido faixa larga. As medidas foram realizadas em um ambiente indoor com freqüência central de $2 \mathrm{GHz}$ e largura de banda de $200 \mathrm{MHz}$. As amplitudes e as fases de 801 freqüiências foram coletadas. Para cada freqüência, o melhor conjunto de antenas foi selecionado. A seletividade espacial foi também avaliada deslocando o receptor MIMO em alguns comprimento de onda da posição inicial. Por fim, a seleção conjunta das antenas e da frequiência foi realizada.
\end{abstract}

Palavras-Chave-Sistemas MIMO, Seleção de Antenas, Medidas de Canal.

Abstract-In this paper we present results regarding antenna selection for a MIMO wideband measurement campaign carried out in indoor scenario with a carrier frequency of $2 \mathrm{GHz}$. The amplitude and phase of the received signal were collected at 801 frequency values in the $200 \mathrm{MHz}$ bandwidth. For each frequency, the best set of antennas was selected. The spatial selectivity was also verified shifting the receiver some wavelengths from the original position. The best antenna antenna subsets and frequency was jointly selected and analyzed for different environments.

Keywords-MIMO Systems, Antenna Selection, Measured Channel.

\section{INTRODUÇÃo}

Sistemas com múltiplas antenas transmissoras e receptoras, também conhecidos como sistemas MIMO (Multiple InputMultiple Output), têm sido apontados como uma solução para aumentar a capacidade e a confiabilidade dos enlaces sem fio, permitindo aos usuários utilizar aplicações com altas taxas de dados [1][2]. Isto é extremamente importante em sistemas onde a capacidade obtida com as técnicas tradicionais é bastante limitada devido às características do ambiente de propagação. Com o sistema MIMO, algumas destas características são exploradas para criar canais paralelos e obter aumento expressivo de capacidade. A análise da capacidade de sistemas MIMO baseia-se em uma modelagem desenvolvida a partir do comportamento estatístico dos pares de enlaces existentes entre as múltiplas antenas transmissoras e receptoras. Existe, portanto, um grande interesse em medir este comportamento para situações típicas bem como em relacionálo a determinados parâmetros do sistema.

Por outro lado, mesmo que a tecnologia MIMO melhore a confiabilidade e as taxas de transmissão, o custo de sua implemetação ainda permanece um desafio. Toda cadeia de transmissão e recepção requer um conjunto de hardwares extras, tais como amplificadores de potência, low noise amplifier

Rodrigo F. Souto e Robson D. Vieira, Instituto Nokia de Tecnologia (INdT), Brasília, Brasil, E-mails: ext-rodrigo.souto@nokia.com, robson.domingos@indt.org.br
(LNA), conversores analógico-digital, entre outros. Assim, a seleção de antenas aparece como uma solução promissora, em que a transmissão/recepção é realizada por meio de um subconjunto das antenas disponíveis, reduzindo o custo de implementação e mantendo grande parte dos benefícios da tecnologia MIMO.

Desenvolver algoritmos que maximizam a diversidade ou a taxa de transmissão têm sido o foco de várias pesquisas. Em [3] e [4], a seleção das antenas para maximizar a diversidade é baseada na norma de Frobenius do canal. Todavia, em [5], a seleção de antenas para maximizar a taxa é baseada na minimização da taxa de erro.

A avaliação dos ganhos de capacidade com seleção de antenas em um canal MIMO real ainda não foi suficientemente analisada. Em [6], a capacidade e a taxa de erro de bits foram avaliadas para um sistema MIMO indoor usando seleção de antenas na frequência central de 5GHz. Em [7], realizou-se uma comparação entre seleção de antenas e seleção de feixe para um ambiente indoor. Desse modo, nosso objetivo é avaliar a seleção de antenas para um canal MIMO faixa larga para diferentes ambientes. Em resumo, este trabalho possui três objetivos principais: (i) avaliar a capacidade do canal com seleção de antenas para um sistema MIMO faixa larga; (ii) analisar o efeito da seletividade espacial no desempenho da selação de antenas e (iii) avaliar a capacidade quando a seleção ótima das antenas e da frequência é realizada.

Este artigo está organizado da seguinte forma: na seção II descreve-se o ambiente de medidas, o processamento dos dados e a análise teórica da capacidade de um canal MIMO. Na Seção III, apresentam-se os resultados das medidas e algumas considerações. Por fim, na Seção IV, têm-se as conclusões do trabalho.

\section{Ambiente e Sistema de Medidas}

\section{A. Ambiente de Medidas}

As medidas foram realizadas na oficina Mecânica do Campus de Engenharia da Universidade Federal Fluminense (UFF). Neste ambiente, o transmissor ficou fixo e receptor foi deslocado para três posições diferentes na oficina. Nestes ambientes foram analisadas situações com visada (ID1), parcialmente com visada (ID2) e sem visada (ID3), conforme ilustrado na Figura 1(a). A oficina mecânica da UFF possui características semelhantes a um ambiente industrial, já que possui pequenos, médios e grandes espalhadores metálicos. Além disso, esta oficina possui praticamente todas as características de outros ambientes, tais como grandes janelas de vidro e objetos de madeiras. 


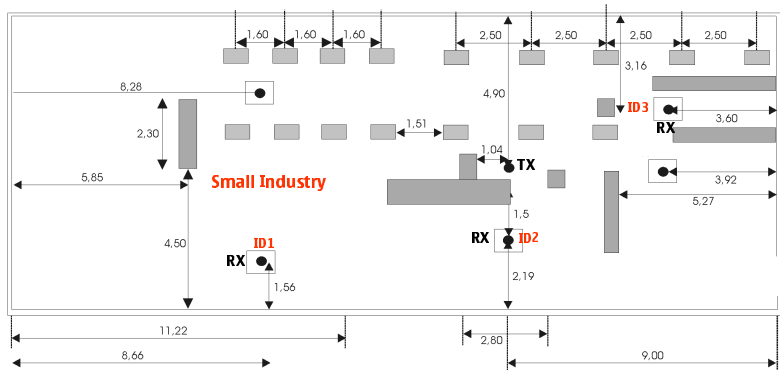

a)

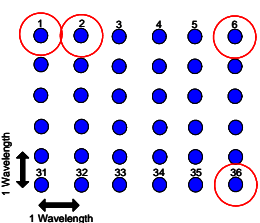

b)

Fig. 1. a) Ambiente da UFF b) Grid 6x6

\section{B. Sistemas de Medidas}

Neste trabalho foi empregada a técnica de sondagem em freqüência para coletar as informações do canal MIMO. Esta técnica pode ser facilmente implementada com o uso de um analisador vetorial, que utiliza uma senóide com amplitude constante para varrer uma determinada faixa de freqüência, investigando, dessa forma, o canal. Para a montagem do sistema de medidas, além do analisador vetorial, foram utilizados os seguintes componentes: dois arranjos de múltiplas antenas, um computador, dois switches, cabos e conectores.

Por meio do analisador vetorial, para cada varredura da banda de interesse, as informações de magnitude e fase foram coletadas. A fim de se obter uma boa resolução temporal, 801 pontos foram selecionados para varrer uma largura de banda de $200 \mathrm{MHz}$ com frequência central em $2 \mathrm{GHz}$.

Dois arranjos lineares foram construídos com seis antenas monopólos de um quarto de comprimento de onda. Porém, apenas quatro elementos foram utilizados para transmissão e recepção, os outros dois elementos inativos, um em cada ponta, foram usados para balancear o efeito do casamento mútuo. Nos dois arranjos, os seis monopólos do receptor e do transmissor eram separados igualmente por meio comprimento de onda $(7,5 \mathrm{~cm})$. Para possibilitar o uso dos arranjos, foram utilizados switches que permitiram o direcionamento dos sinais.

Com o objetivo de se obter uma varredura completa da banda de interesse, o analisador enviava um sinal por um cabo coaxial de $50 \mathrm{~m}$ até o arranjo das antenas transmissoras. $\mathrm{Na}$ transmissão, o switch direcionava os sinais nas antenas transmissoras, enquanto que na recepção, o sinal que chegava no arranjo era direcionado pelo uso de outro switch para um amplificador de baixo ruído (LNA) que, por sua vez, enviava as informações para o analisador vetorial. $\mathrm{O}$ analisador enviava os dados coletados por meio de um barramento HPIB para o computador, que os armazenava.

Conforme já citado, para a caracterização do canal foi necessário o uso de cabos e conectores para interligar os arranjos de transmissão e recepção ao analisador. Estes componentes, apesar de estarem no sistema de medição, não devem influenciar na medida. Assim, para garantir uma medida correta, o procedimento de calibração fora realizado como descrito em [8] e [9].

\section{Seleção de Antenas e Processamento de Dados}

\section{A. Seleção de Antenas}

Um canal de rádio faixa estreita pode ser caracterizado por um ganho que não varia ao longo da faixa. Fazendo esta consideração para os diversos canais de um sistema MIMO, pode-se escrever que o sinal recebido na antena receptora $i$ correspondente a um sinal $s_{j}(t)$ na antena transmissora $j$ será dado por $G_{i}(t) s_{j}(t)$, onde $G_{i}(t)$ é o ganho do sub-canal entre a antena transmissora $j$ e a antena receptora $i$. Considerando o conjunto de $N$ antenas transmissoras e $M$ antenas receptoras, o sinal recebido na antena receptora $i$ será

$$
r_{i}(t)=\sum s_{j}(t) G_{i, j}+n_{i}(t), j=1 \ldots N, i=1 \ldots M
$$

onde $n_{i}(t)$ é um ruído branco gaussiano na saída da antena receptora $i$.

A capacidade do canal é um dos parâmetros mais importantes no desempenho de um sistema de transmissão e estabelece o limite na taxa de bits que pode ser transmitida por meio do canal. Para um sistema MIMO, e considerando que o transmissor não tem informação sobre a matriz de canal, a capacidade de um canal MIMO é dada por

$$
C=\log _{2}\left|I_{N}+\frac{\rho}{M} H H^{H}\right| \text { bits } / s / H z,
$$

onde $\mathbf{I}_{N} \in \Re^{N \times N}$ é uma matriz identidade, $\rho$ é a razão sinalruído média (SNR) recebida em cada antena receptora e $H$ é a matriz normalizada determinística do canal.

A partir dos arranjos de 4 antenas de transmissão e recepção, o melhor subconjunto de 2 e 3 antenas para o transmissor e receptor foi selecionado conjuntamente. O critério de seleção foi a capacidade do canal dada pela Eq. 2.

Para cada ambiente medido, posição do grid e freqüência, a capacidade pode ser maximizada. A função objetivo pode ser escrita como

$$
\max C_{p, f}\left(\mathbf{H}_{s}\right)
$$

para todo $p$ e $f$, em que $p$ é a posição no grid, $f$ é a freqüência e $\mathbf{H}_{s} \in \Im^{N \times M}$ é a matriz do canal do subconjunto.

Para um canal seletivo na frequiência, diferentes valores de capacidade podem ser obtidos para as diferentes freqüências. Assim, para cada ambiente e posição no grid, pode-se selecionar de forma conjunta o melhor subconjunto de antenas e a melhor freqüência. Para a seleção conjunta de antenasfreqüência, a Eq. 3 pode ser redefinida como

$$
\max C_{p}\left(\mathbf{H}_{s}, f\right)
$$

para todo $p$. 


\section{B. Metodologia e Processamento de Dados}

As medidas foram realizadas sobre um grid quadrado horizontal, com 36 pontos separados por um comprimento de onda, como mostrado na Figura 1. As alturas do transmissor e receptor foram fixadas a $1,5 \mathrm{~m}$ do chão. Para cada ponto do grid, coletaram-se as amplitudes e fases do sinal recebido para as 801 frequiências de uma banda de $200 \mathrm{MHz}$. Entretanto, apenas quatro posições (P1, P2, P6 e P36) no grid foram escolhidas para serem apresentadas neste estudo. Estas posições estão marcadas com um círculo na Figura 1(b).

A partir dos arranjos de 4 antenas de transmissão e recepção, 36 e 16 subconjuntos são possíveis quando se considera a seleção 2 e 3 antenas respectivamente. A avaliação da capacidade e da seleção de antenas foram feitas diretamente dos dados medidos. As Tabelas I e II apresentam os subconjuntos possíveis para sistemas com 2 e 3 antenas respectivamente.

TABELA I

SUBCONJUNTOS DISPONÍVEIS COM 2 ANTENAS .

\begin{tabular}{|c|cccccc|}
\hline Receptores & \multicolumn{6}{|c|}{ Subconjuntos de Transmissores } \\
\cline { 2 - 7 } & $\{1,2\}$ & $\{1,3\}$ & $\{1,4\}$ & $\{2,3\}$ & $\{2,4\}$ & $\{3,4\}$ \\
\hline$\{1,2\}$ & 1 & 7 & 13 & 19 & 25 & 31 \\
$\{1,3\}$ & 2 & 8 & 14 & 20 & 26 & 32 \\
$\{1,4\}$ & 3 & 9 & 15 & 21 & 27 & 33 \\
$\{2,3\}$ & 4 & 10 & 16 & 22 & 28 & 34 \\
$\{2,4\}$ & 5 & 11 & 17 & 23 & 29 & 35 \\
$\{3,4\}$ & 6 & 12 & 18 & 24 & 30 & 36 \\
\hline
\end{tabular}

TABELA II

SubCONJUNTOS DISPONÍVEIS COM 3 ANTENAS

\begin{tabular}{|c|cccc|}
\hline Receptores & \multicolumn{4}{|c|}{ Subconjunto de Transmissores } \\
\cline { 2 - 5 } & $\{1,2,3\}$ & $\{1,2,4\}$ & $\{1,3,4\}$ & $\{2,3,4\}$ \\
\hline$\{1,2,3\}$ & 1 & 5 & 9 & 13 \\
$\{1,2,4\}$ & 2 & 6 & 10 & 14 \\
$\{1,3,4\}$ & 3 & 7 & 11 & 15 \\
$\{2,3,4\}$ & 4 & 8 & 12 & 16 \\
\hline
\end{tabular}

\section{Resultados}

\section{A. Cálculo de Capacidade}

Para o cálculo da capacidade, utilizou-se a Eq. 2 e normalizaram-se os ganhos do canal para diferentes valores de SNR. Além disso, a fim de se avaliar a capacidade com a seleção de antenas, quatro casos de referências foram definidos. No primeiro caso, uma antena do transmissor e uma do receptor foram escolhidas e mantidas fixas para todos ambientes, pontos do grid e freqüências. O subconjunto é formado pela antena 1 do transmissor e pela antena 1 do receptor. Da mesma forma, nos segundo e terceiro casos, 2 e 3 antenas no transmissor e receptor foram escolhidas e mantidas fixas. $\mathrm{O}$ subconjunto 1 foi escolhido como referência para o caso com duas antenas, conforme Tabela I. Para o estudo com três antenas, o subconjunto 1 foi escolhido como referência, segundo a Tabela II. E no último caso, todas as antenas foram consideradas. Nas figuras a seguir, SO representa os casos de seleção de antenas e Ref, os casos de referência. O critério de seleção é dado pela Eq. 3 .

As Figuras 2, 3 e 4 apresentam as capacidades do canal MIMO com a seleção de antenas e o casos de referência para as quatro posições do grid (P1, P2, P6 e P36) e para a freqüência central de $2 \mathrm{GHz}$.
Para o ambiente com visada direta (ID1), a seleção com 3 antenas obteve quase a mesma capacidade da configuração de 4 antenas. Neste caso, em algumas posições do grid, ganhos maiores que $1 \mathrm{bit} / \mathrm{s} / \mathrm{Hz}$ só foram observados para valores de SNR acima de $25 \mathrm{~dB}$. Para todas as posições observadas, a seleção com 3 antenas obteve uma capacidade maior do que a configuração de referência com 3 antennas. Ganhos em tornor de 0.5 a 3.2 bits $/ \mathrm{s} / \mathrm{Hz}$ foram obtidos.

Para o caso de seleção com 2 antenas, as capacidades obtidas foram maiores que para os casos de referência com 2 e 3 antenas, quando os valores de SNR eram inferiores a $20 \mathrm{~dB}$ para este último. Novamente, os ganhos variaram de acordo com a posição no grid (aproximadamente de 1.6 a 3 bits/s/Hz), mostrando, dessa forma, o efeito da seletividade espacial na capacidade do canal. Por exemplo, as posições P1 e P2 são separadas por 1 comprimento de onda $(15 \mathrm{~cm})$ e a capacidade diminuiu em 2 e 0.5 bits $/ \mathrm{s} / \mathrm{Hz}$ (comparando com os casos de referência) com a seleção de antenas para 2 e 3 antenas respectivamente. Entretanto, para as posições P6 e P36, que estão separadas por 5 comprimentos de onda, uma redução de 1 bit/s/Hz foi obtida com seleção de antenas com 3 antenas e um aumento de $0.5 \mathrm{bits} / \mathrm{s} / \mathrm{Hz}$ foi obtido com seleção de 2 antenas.

Para o ambiente com visada parcial (ID2), a seleção de 2 e 3 antenas apresentaram diferentes desempenhos ao longo do grid de medidas, uma vez que, dependendo da posição, algumas antenas possuíam visada e outras não. Na Figura 3, a seleção com 3 antenas não apresentou ganhos significativos em relação ao caso de referência com 3 antenas. Porém, tanto o caso de referência (3 antenas) como a seleção de 3 antenas apresentaram aproximadamente a mesma capacidade que a configuração com 4 antenas. Por outro lado, para a seleção com 2 antenas, e para a maioria das posições do grid, ganhos significativos (de 1.0 a $4.6 \mathrm{bits} / \mathrm{s} / \mathrm{Hz}$ ) foram obtidos. Neste ambiente, o efeito da seletividade espacial fica mais evidente, exercendo maior influência na seleção com 3 antenas.

No ambiente sem visada (ID3), como observado nos outros ambientes, a seleção com 3 antenas apresentou capacidades próximas às obtidas com a configuração de 4 antenas. Ganhos significativos (de 1.4 a 2.8 bits $/ \mathrm{s} / \mathrm{Hz}$ ) só foram observados para valores de SNR maiores que $25 \mathrm{~dB}$. Por outro lado, para a maioria das posições do grid, foram observadas pequenas variações, aproximadamente 2 bits $/ \mathrm{s} / \mathrm{Hz}$, nos ganhos com seleção de 2 antenas. Novamente, o desempenho da seleção de antenas foram influenciados pela seletividade espacial.

Em todos os ambientes medidos, observou-se que os recursos espaciais (as antenas) podem não ser bem utilizados. Em quase todos os casos, a seleção com 3 antenas apresentou quase a mesma capacidade que a configuração com 4 antenas e foi melhor ou igual ao caso de referência com 3 antenas, indicando o desperdício de recurso. Do mesmo modo, a seleção com 2 antenas, em vários casos, apresentou aproximadadmente o mesmo desempenho que o caso de referência com 3 antenas e foi melhor ou igual ao caso de referência com 2 antenas.

As Figuras 5, 6 e 7 mostram as capacidades calculadas para todas as frequiências com um SNR de $20 \mathrm{~dB}$. Observase que, para algumas freqüências, as capacidades obtidas com a seleção de 3 antenas e o caso de referência com 4 antenas 

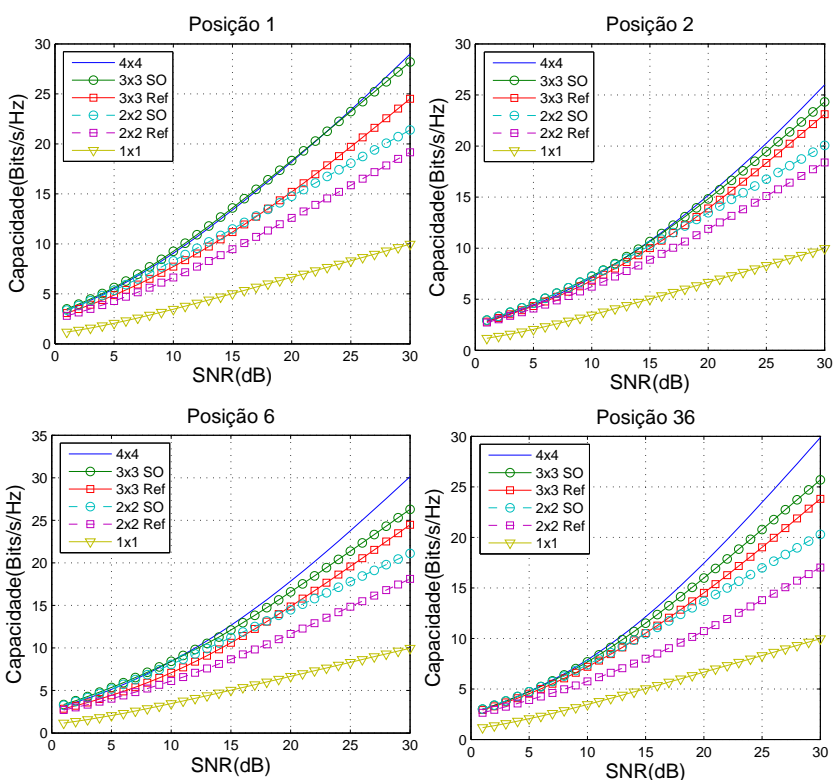

Fig. 2. Capacidade com seleção de antenas para o ambiente ID1
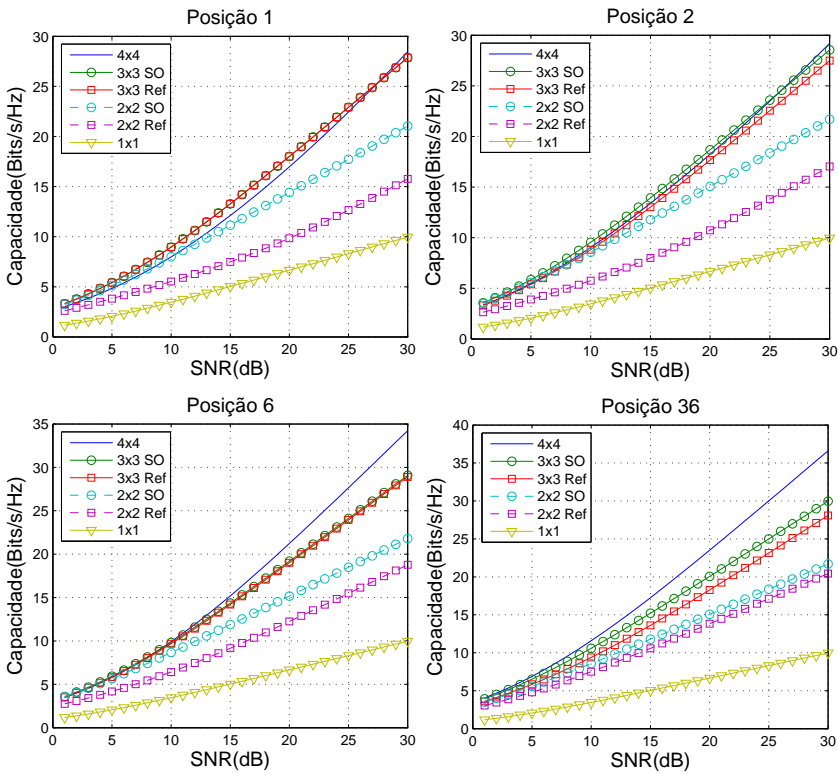

Fig. 3. Capacidade com seleção de antenas para o ambiente ID2.

são bem próximas. $\mathrm{O}$ mesmo ocorreu para o caso de seleção de 2 antenas e o caso de referência com 3 antenas. Para todas as freqüências, as capacidades obtidas com a seleção de antenas foram maiores que os casos de referências (exceto quando se compara com o caso de referência com 4 antenas).

É possível notar uma grande variação na capacidade ao longo da banda para os casos de referência com 2 e 3 antenas. Observam-se, por exemplo, reduções de até 5 bits $/ \mathrm{s} / \mathrm{Hz}$ na capacidade. Porém, com a seleção de antenas essa variação é reduzida. A Tabela IV apresenta a capacidade média e a condicionada para todas as freqüências para os casos de referências e de seleção.

Para o caso com 3 antenas, a melhoria obtida com a seleção nas capacidades média e condicionada foi bastante significativa. Em alguns casos, ganhos maiores que $3 \mathrm{bits} / \mathrm{s} / \mathrm{Hz}$ foram obtidos na capacidade condicionada. Já para os casos com 2 antenas, além dos ganhos nas capacidades média
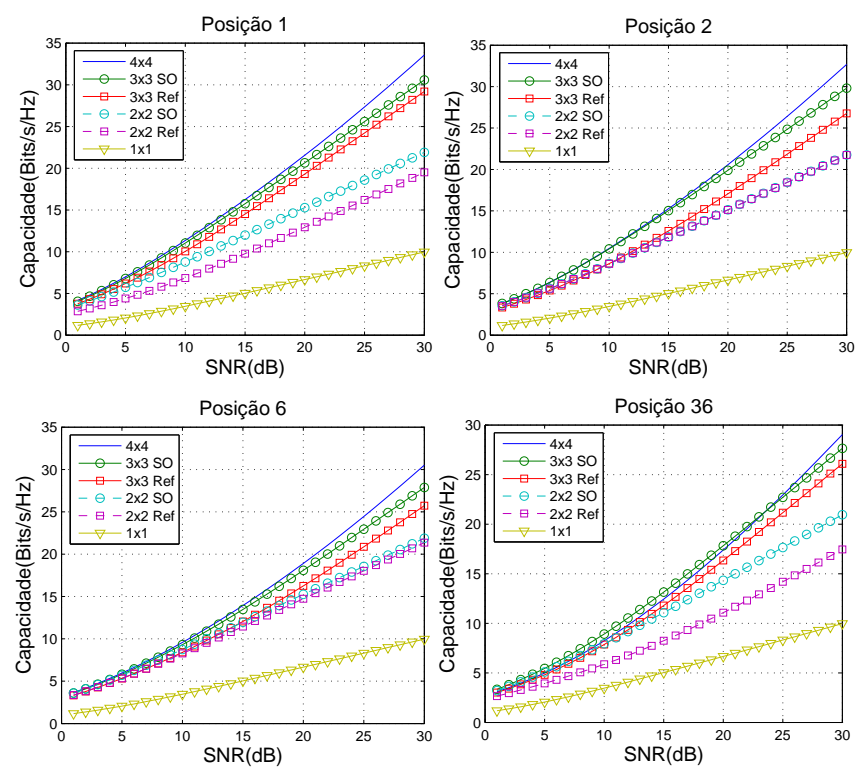

Fig. 4. Capacidade com seleção de antenas para o ambiente ID3.

TABELA III

CAPACIDADES MÉDIA E CONDICIONADA PARA CONFIGURAÇÕES COM 3 ANTENAS.

\begin{tabular}{|c|c|c|c|c|}
\hline \multirow{3}{*}{ Ambiente } & \multicolumn{4}{|c|}{ Capacidade $[$ bits $/ \mathrm{s} / \mathrm{Hz}]$} \\
\hline & \multicolumn{2}{|c|}{ Antenas Fixas } & \multicolumn{2}{|c|}{ Seleção de Antenas } \\
\hline & Média & Condicionada & Média & Condicionada \\
\hline ID1 & 15.6 & 13.5 & 17.7 & 16.0 \\
\hline ID2 & 16.8 & 14.3 & 18.3 & 16.2 \\
\hline ID3 & 18.5 & 16.4 & 19.8 & 18.8 \\
\hline
\end{tabular}

TABELA IV

CAPACIDADES MÉDIA E CONDICIONADA PARA CONFIGURAÇÕES COM 2 ANTENAS.

\begin{tabular}{|c|c|c|c|c|}
\hline \multirow{3}{*}{ Ambiente } & \multicolumn{4}{|c|}{ Capacidade $[$ bits $/ \mathrm{s} / \mathrm{Hz}]$} \\
\hline & \multicolumn{2}{|c|}{ Antenas Fixas } & \multicolumn{2}{|c|}{ Seleção de Antenas } \\
\hline & Média & Condicionada & Média & Condicionada \\
\hline ID1 & 12.0 & 10.9 & 14.7 & 13.9 \\
\hline ID2 & 12.6 & 11.0 & 14.8 & 14.0 \\
\hline ID3 & 13.8 & 11.8 & 15.2 & 15.0 \\
\hline
\end{tabular}

e condicionada, houve também uma aproximação entre a capacidade condicionada e a média.

A Figura 8 apresenta as capacidades para seleção conjunta das antenas e de frequência (SOFreq), seleção de antenas para uma frequiência fixa $(2 \mathrm{GHz})$ e os casos de referência. Neste caso, o critério de seleção de antenas é dado pela Eq. 4.

A seleção conjunta das antenas e de freqüência para o caso de 2 antenas quase não mostrou ganhos na capacidade. Conforme explicado anteriormente, com a seleção de antenas houve uma redução na variação da capacidade ao longo das freqüências e, para o caso de 2 antenas, a capacidade condicionada ficou bem próxima à capacidade média. Por este motivo, são esperadas pequenas variações na capacidade para as diferentes frequiências. Por outro lado, para o caso de 3 antenas, ganhos foram obtidos quando a seleção foi realizada em conjunto com a freqüência. Porém, a seletividade espacial pode ainda reduzir ou aumentar estes ganhos.

\section{Conclusões}

Neste trabalho, apresentaram-se os resultados da seleção de antenas para um canal MIMO de faixa larga obtidos 


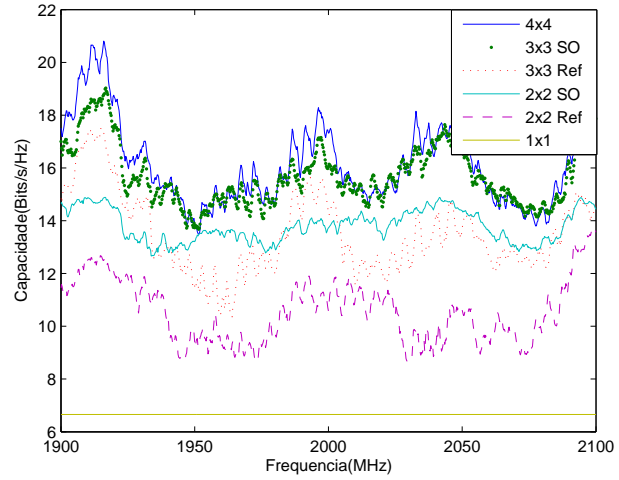

Fig. 5. Variação da capacidade por frequência para o ambiente ID1.

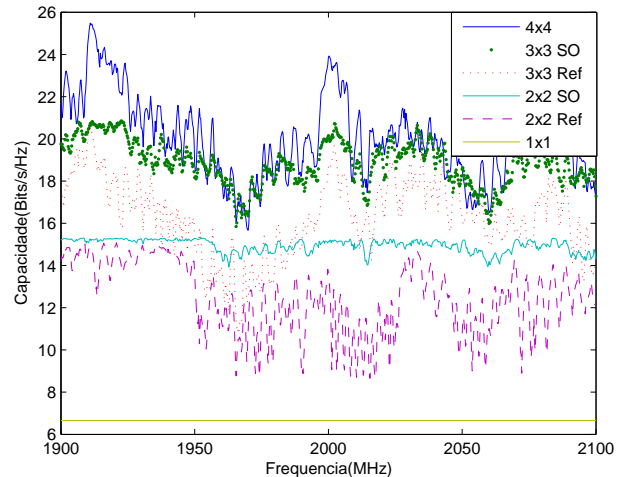

Fig. 6. Variação da capacidade por frequência para o ambiente ID2.

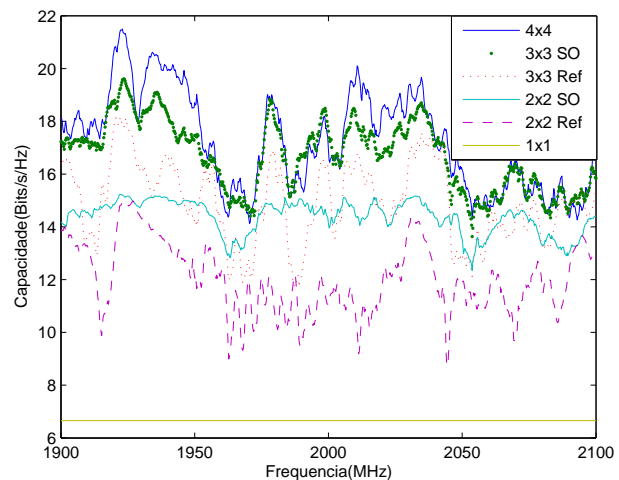

Fig. 7. Variação da capacidade por frequência para o ambiente ID3.

em diferentes ambientes indoor. Para todos os ambientes, as capacidades obtidas com seleção com 2 e 3 antenas foram próximas às capacidades dos casos de referência com 3 e 4 antenas respectivamente. Com a seleção de antenas, as variações da capacidade ao longo de uma banda de transmissão foram reduzidas e as capacidades médias e condicionadas melhoradas.

Os resultados mostraram os benefícios que podem ser obtidos com seleção de antenas, impedindo o desperdício de recursos espaciais. Tais informações podem ser utilizadas, por exemplo, no desenvolvimento de novos algoritmos de escalonamentos.

\section{AgRAdecimentos}

Os autores agradecem ao Instituto Nokia de Tecnologia (INdT) pelo apoio recebido.
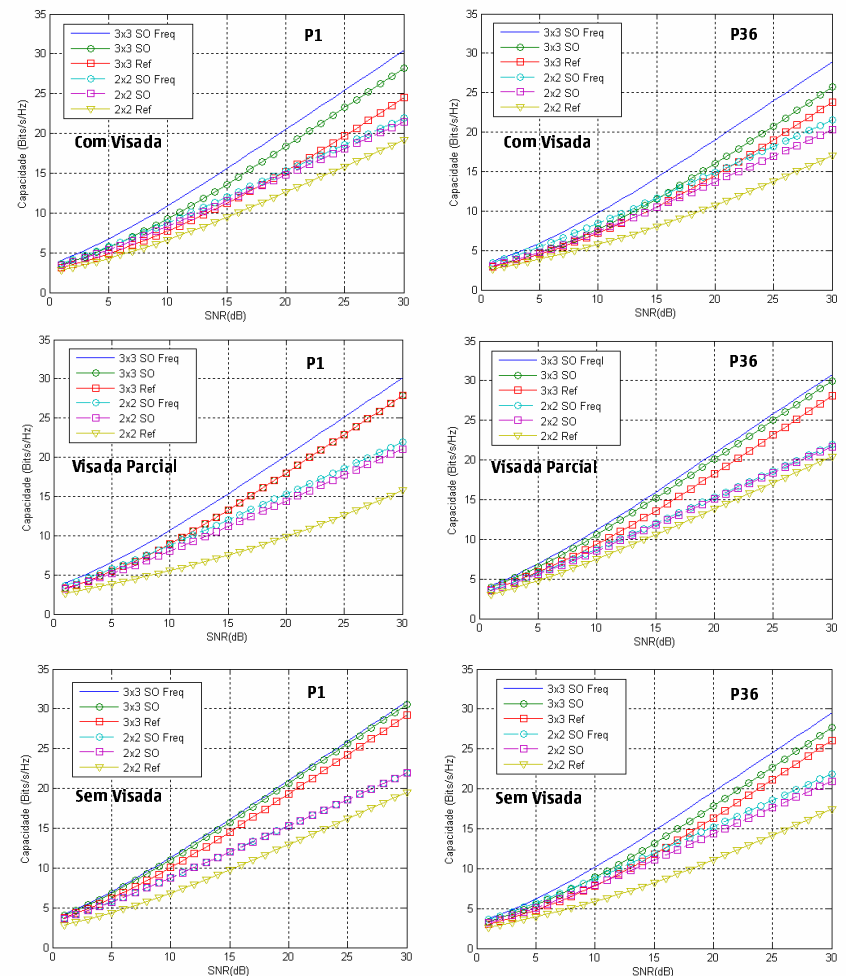

Fig. 8. Seleção de antenas-frequência

\section{REFERÊNCIAS}

[1] G. J. Foschini and M. J. Gans, "On Limits of Wireless Communications in a Fading Environment when Using Multiple Antennas," Wireless Personal Communications, vol. 06, no. 03, 1998.

[2] G. J. Foschini, "Layered Space-Time Architecture for Wireless Communications in a Fading Environment when using Multiple Antennas," Bell Labs Tech. J., no. 2, 1996.

[3] D.Gore and A.J.Paulraj, "MIMO Antenna sub-set Selection for SpaceTime Coding," IEEE Transactions on Signal Processing, no. 50, 2002.

[4] M. A.F. Molisch and J.Winters, "Reduced-complexity transmit/receivediversity System," IEEE Transactions on Signal Processing, no. 51, 2003.

[5] R. Heath and A.J.Paulraj, "Antenna Selection for Spatial-Multiplexing System based on Minimum Erro Rate," in IEEE International Conference on Communications, vol. 7, 2001.

[6] I. I. S. H. Y. Nakaya, A. Honda and Y. Oishi, "Measured Capacity Evaluation of Indoor Office MIMO Systems using Receive Antenna Selection," in IEEE Vehicular Technology Conference, vol. 6, 2006.

[7] J. Jiang and M. Ingram, "Comparison of Beam Selection and Antenna Selection Techniques in Indoor MIMO Systems at $5.8 \mathrm{ghz}$," in IEEE Radio and Wireless Conference, 2003, pp. 179-182.

[8] R.D.Vieira and G.L.Siqueira, "MIMO Measured Channels: Capacity Results and Analysis of Channel Parameters," in IEEE International Telecommunications Symposium, 2006.

[9] R.D.Vieira, "Medidas do Canal MIMO Indoor: Analise da Capacidade e dos Parametros do Canal," Ph.D. dissertation, Pontificia Universidade Catolica do Rio de Janeiro (PUC-Rio), 2005.

[10] D. H.Bolcskei and A.J.Paulraj, "Impact of Propagation Environment on the Performance of Space-Frequency Coded MIMO-OFDM," IEEE Journal on Selected Areas in Communications, no. 21, 2003.

[11] _ "On the Capacity of OFDM based Spatial Multiplexing Systems," IEEE Transactions on Communications, no. 50, 2002. 